\title{
ESTUDIO DE PRODUCTOS FORESTALES NO MADEREROS (PFNM) EN LA REGIÓN DE AYSÉN, CHILE
}

\author{
Salinas, J. ${ }^{1}$; Moya, I. ${ }^{1}$ y Gómez, C. ${ }^{2}$
}

\section{RESUMEN}

Los bosques nativos de Chile cubren una superficie de más de 13 millones de hectáreas y de esta superficie cerca de un $32 \%$ se encuentra en la Región de Aisén. Estos bosques albergan una amplia variedad de Productos Forestales no Madereros (PFNM), que han sido utilizados históricamente por las poblaciones indígenas y campesinas, destacando productos con potencial médico, alimenticio, decorativo y de abrigo.

Este tipo de productos secundarios de los bosques es de importancia para las comunidades rurales, que los recolectan para su propio consumo y para comercializarlos, y han estado adquiriendo una particular relevancia dada por el creciente interés de los mercados nacionales y externos por productos naturales, en especial por aquellos de carácter alimenticio, como hongos, frutos y otros. Las exportaciones chilenas de estos productos están en aumento anualmente y alcanzan ya a unos US $\$ 80$ millones.

Se estima que actualmente en el país estos productos generan más de 200 mil empleos en zonas rurales, con una marcada connotación de género y un efecto de retención de población rural que aminora la migración de esta a los centros urbanos. La recolección de estos productos en Aisén es una actividad silenciosa, no reconocida en los circuitos productivos locales, y de bajo desarrollo tecnológico. No hay programas que fomenten y controlen la actividad y sus prácticas de cosecha, por lo que se pone en riesgo la sostenibilidad de algunos de estos recursos, y existen algunos poderes de compra esporádicos y foráneos que provocan distorsión de precios a nivel de monopsonio. Sin embargo, se percibe un gran potencial en la puesta en valor estos productos como bienes adicionales generados en los ecosistemas forestales.

El objetivo del presente trabajo fue realizar un estudio de los PFNM en la Región de Aisén y constituye un primer acercamiento a los actores dedicados a la actividad para visualizar los principales recursos y los productos con mayor potencial económico. El estudio se concretó a través de la aplicación de encuestas y entrevistas a personas que se dedican a la actividad de recolección y comercialización de PFNM, reuniendo información sobre los recursos recolectados y las prácticas de generación de valor asociadas. Para el análisis de la información se elaboraron matrices sistematizadas para la obtención de frecuencias y medidas de variabilidad, utilizando un enfoque comparativo entre variables.

Los resultados obtenidos, indican que la actividad de recolección, dentro de la diversidad de labores que se efectúa en el espacio rural, genera sobre el $20 \%$ del ingreso total en más de un tercio de la población entrevistada. Es una actividad desarrollada esencialmente por mujeres, en el $87 \%$ de los casos, y el aprendizaje se origina principalmente en la familia (45\%).

Los productos que poseen mayor potencial comercial en la región son maqui, rosa mosqueta, calafate y morchella. Sin embargo, en la región hay un largo listado de PFNM identificados y otros que no fueron posibles catastrar.

Palabras claves: Patagonia chilena, Productos forestales no madereros, Maqui, Morchella, Rosa mosqueta.

\footnotetext{
${ }^{1}$ Ingenieros Forestales, Instituto Forestal, Sede Patagonia, Coyhaique, Chile. jsalinas@infor.cl; imoya@infor.cl

${ }^{2}$ Consultora privada, Coyhaique, Chile. cgnomenn@gmail.com
} 


\section{SUMMARY}

Native forests in Chile cover over than 13 million hectares and about $32 \%$ of this area corresponds to the Aisén Region. Associated to the native forests there is a wide variety of Non Wood Forest Products (NWFP), which have historically been used by indigenous and peasant farmer communities, especially products with medicinal, food, decorative or shelter potential.

These secondary forest products are important to rural communities which collect them for covering theirs own needs or for sale, and are gaining a particular relevance because of the increasing interest in local and foreign markets for natural products, especially those related to foods, such as fungus, fruits and others. Chilean exports of this kind of products are annually increasing and are already close to US $\$ 80$ million.

At the national level it is estimated that at present NWFP are generating over than 200 thousand employments in rural areas, with high women participation and a good effect on reducing migration to urban centers. NWFP collection in Aisén is a quiet activity, not recognized in the local production sphere. There are no promotion and control programs, current harvesting practices represent a risk to products sustainability, and there are some occasional and foreign buyers who distort the prices at monopsony level. However, a great potential is foreseen in the valuation of these products as additional native forest goods.

The research main objective is a review of NWFP in the region through a first approach to the stakeholders to identify the main resources and those with a greater economic potential. The study was developed through direct surveys to stakeholders, to gather information on collected resources and the associated add value practices. Systematized matrixes were elaborated to obtain frequencies and variability measures using a variables comparative standpoint.

The results show that the collection activity, under the frame of the variety of activities developed in the rural sphere, generates over than $20 \%$ of the total income to more than one third of the survey respondents, is a work developed mainly by women $(87 \%)$ and the learning mainly comes from the family (45\%).

Products with the higher economic potential are Maqui, Rosa Mosqueta and Morchella, but there are in the Region a number of identified NWFP and others which was not possible to register.

Key words: Chilean Patagonia, Non Wood Forest Products, Maqui, Morchella, Rosa mosqueta. 


\section{INTRODUCCION}

Los Productos Forestales No Madereros (PFNM), conocidos internacionalmente como Non Timber Forest Products (NTFP) o Non Wood Forest Products (NWFP), corresponden a una denominación comúnmente utilizada para un sector de la producción forestal que todavía no tiene una terminología consensuada. FAO (1999) los define como "bienes de origen biológico, distintos de la madera, derivados del bosque, de otras áreas forestales y de los árboles fuera de los bosques".

Los bosques nativos de la Región de Aisén representan cerca del 32\% de la superficie de bosques nativos de Chile, según la última actualización del Catastro del Bosque Nativo. En ellos se encuentran diferentes Productos Forestales no Madereros (PFNM), como maqui, frutilla silvestre, líquenes, follaje, coligue, rosa mosqueta, calafate, hongos comestibles, hierbas de uso medicinal o tintóreo, flora melífera, y otros, lo que da cuenta de una riqueza no reconocida formalmente en los circuitos productivos locales, pero muy valorada en las zonas rurales.

Durante la temporada 2013, este rubro genera al país un ingreso anual de US $\$ F O B$ 80,1 millones, aumentando un 7,5\% respecto al monto obtenido durante el año anterior (INFOR, 2014). Se mantienen crecimientos promedio anuales en torno al $9 \%$ en las últimas dos décadas y se amplían sustancialmente los mercados de destino, posicionando la recolección de PFNM como una actividad demandante de mano de obra, con alta connotación de género y que contribuye a la retención de la población rural.

La recolección de PFNM en la Región de Aisén es una actividad fuertemente arraigada en las localidades rurales. Si bien posee obvias implicancias económicas para las familias y sus ingresos, se carecía de conocimiento en la región sobre el desarrollo de los recursos naturales involucrados, las características de los procesos de producción y comercialización, y las potencialidades de crecimiento.

La importancia de valorizar los PFNM, ha hecho cambiar el paradigma sobre la función del bosque nativo, que ya no puede ser únicamente la generación de productos maderables, y esto motivó la elaboración del presente trabajo, que busca conocer la actividad de recolección y los productos que se recolectan para dar una valoración extra a este recurso de la Patagonia de Aisén.

\section{OBJETIVO}

El objetivo del presente trabajo fue estudiar y catastrar los Productos Forestales no Madereros (PFNM) presentes en la Región de Aisén, a través de un estudio descriptivo, constituyendo el primer acercamiento a los actores dedicados a la actividad de recolección.

\section{METODOLOGIA}

El presente trabajo se enmarca en el paradigma de investigación post-positivista y corresponde a un estudio cuantitativo, de carácter exploratorio, que rescata información de los Productos Forestales No Madereros de la Región de Aisén, y constituye un primer acercamiento a los actores relacionados con la recolección, producción y comercialización de estos productos, representando parte importante de su cadena productiva.

\section{Levantamiento de Información}

El estudio rescató información en la mayoría de las localidades y comunas de la Región de Aisén, de acuerdo a una serie de etapas secuenciales que son descritas a continuación:

\section{-Elaboración de Encuesta}

El equipo técnico de INFOR sede Patagonia elaboró una encuesta, con el apoyo del Instituto Nacional de Estadística (INE), que fue consensuada con profesionales integrantes de la 
Mesa Publica de PFNM de la Región de Aisén.

\section{-Reunión-Taller}

Con profesionales de instituciones del AGRO y Municipalidades, el equipo sostuvo reuniones en las distintas localidades de la región, con el fin de entregar información y capacidades sobre los PFNM a funcionarios de INDAP, CONAF, SAG y PRODESAL, hacerlos participes del estudio y establecer un marco de cooperación para el levantamiento de información. Dada la información sobre los temas generales en torno a los PFNM, se da inicio a un proceso de sociabilización de la encuesta, para lo cual se planificó un taller de trabajo que permitió conocer los detalles de esta.

\section{-Reunión con la Comunidad}

El objetivo de esta actividad fue convocar a personas interesadas, que además trabajan con PFNM. Para la convocatoria se realizó avisos radiales e invitaciones dirigidas. Esta reunión incluyó una presentación técnica, que abordó los conocimientos generales y posteriormente las estadísticas y el mercado de PFNM, los principales productos presentes en la región y sus usos, para finalmente entrevistar a los recolectores y obtener los primeros antecedentes respecto de la población objetivo.

\section{Procesamiento de Datos y Análisis de la Información}

Terminada la etapa de recopilación de la información, las encuestas fueron ordenas e ingresadas a una base de datos digital. Se procedió a analizarlas de dos formas de acuerdo al tipo de pregunta que se tratase.

En el caso de las preguntas cerradas, se elaboró matrices de datos sistematizadas en planillas de formato Microsoft Excel, con el fin de filtrar los campos de interés, y se obtuvo los resultados de frecuencias para cada una de ellas. Dependiendo del tipo de datos, se consideró también medidas de variabilidad.

Los resultados de las preguntas abiertas fueron ordenados de acuerdo a la estructura general de clasificación de PFNM, propuesta para el país. El análisis de los datos se realizó mediante un enfoque comparativo, el cual se presenta en los comentarios y observación a cada variable.

\section{RESULTADOS}

\section{Antecedentes Generales de los Recolectores}

Los potenciales entrevistados, derivaron en su totalidad de pobladores de localidades rurales y fueron contactados según avanzaba el estudio. A las personas que finalmente constituyeron el grupo objetivo se las caracterizó según los rasgos que se indica continuación.

\section{-Composición por Género}

Se entrevistó un total de 105 personas, las cuales en su mayoría fueron mujeres (87\%), lo cual se explica por el hecho que la actividad de recolección, dentro de la diversidad de actividades que se efectúa en el espacio rural, es una actividad esencialmente femenina. La participación de hombres en labores de recolección represento un $13 \%$, demostrando que hay prioridad por otras actividades productivas, como la ganadería y leña principalmente. 


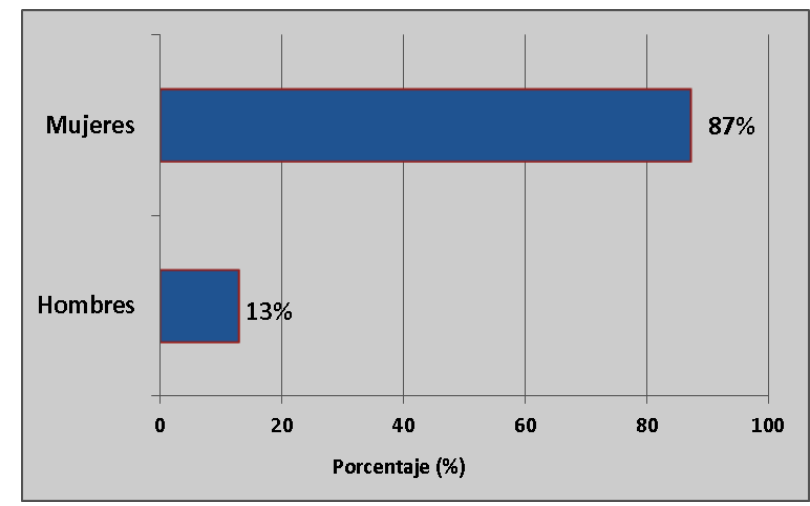

Figura $\mathrm{N}^{\circ} 1$

COMPOSICIÓN POR GÉNERO DE PERSONAS ENTREVISTADAS

\section{-Ubicación Geográfica}

El grupo de recolectores entrevistados se encuentra distribuido principalmente en siete comunas de la región. Sin embargo, se estima que existe una participación de recolectores en la totalidad de comunas de la región. La comuna con mayor presencia de recolectores $(35,2 \%)$ fue Cochrane, seguida por Aisén y Río lbáñez con $20 \%$ cada una.

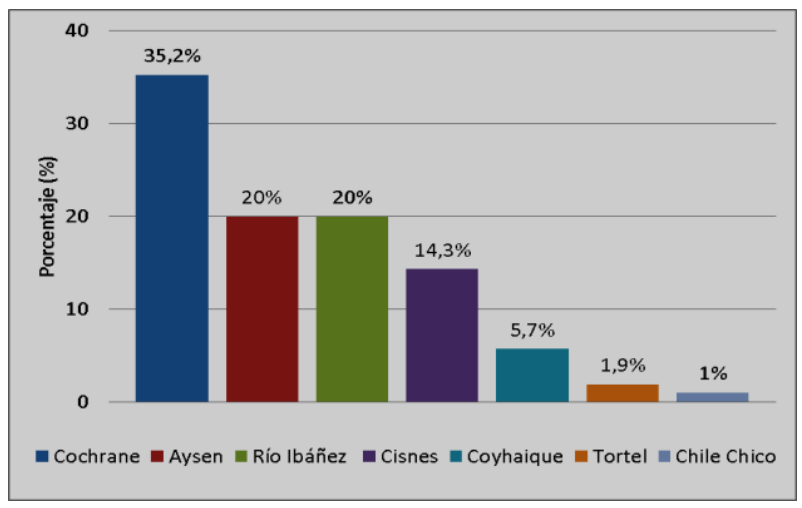

Figura $\mathrm{N}^{\circ} 2$

PARTICIPACIÓN PORCENTUAL DE RECOLECTORES POR COMUNA

\section{-Rango Etáreo}

Al agrupar a los recolectores en rangos de cerca de 10 años edad, se aprecia que un porcentaje significativo (37\%) está dado por jóvenes o adultos jóvenes. Información de importancia ya que esta situación tiene consecuencias, tanto en la proyección de la actividad como en la sustentación para la creación de programas que impulsen su desarrollo, asignando recursos para tal efecto. El 50\% de los entrevistados se encuentra entre los $40-60$ años, situación que respalda el aprendizaje y mantención de conocimiento entregado por prosapia. 


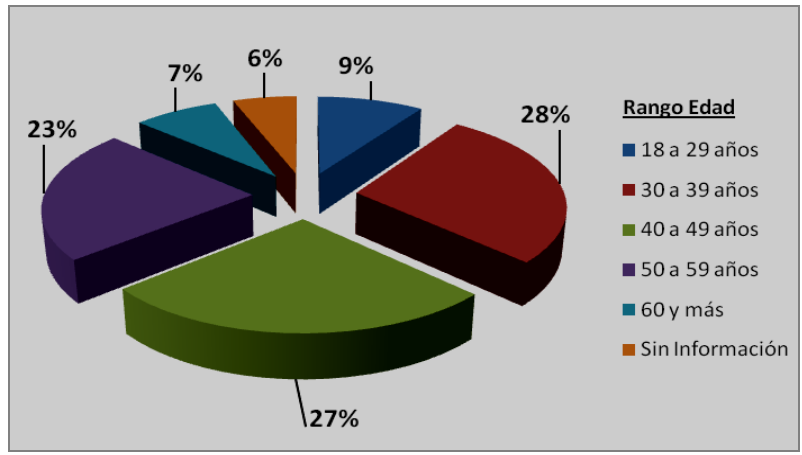

Figura $\mathrm{N}^{\circ} 3$

COMPOSICIÓN DE ENCUESTADOS POR RANGO DE EDAD

\section{-Nivel de Escolaridad}

La información obtenida indica un bajo nivel de escolaridad. Del total de personas entrevistadas, un $59 \%$ posee solo educación básica. Al mismo tiempo llama la atención que el 3\% presenta formación universitaria y se dedica a actividades relacionadas con PFNM.

Cuadro $\mathrm{N}^{\circ} 1$

NIVEL DE ESCOLARIDAD DE PERSONAS ENTREVISTADAS

\begin{tabular}{|l|c|c|}
\hline \multirow{2}{*}{ Variables } & \multicolumn{2}{|c|}{ Entrevistados } \\
\cline { 2 - 3 } & (No) & (\%) \\
\hline Educación Básica & 62 & 59 \\
Educación Media & 30 & 29 \\
Educación Técnica & 6 & 5 \\
Educación Universitaria & 3 & 3 \\
Sin Educación Formal & 2 & 2 \\
Sin Información & 2 & 2 \\
\hline Total & $\mathbf{1 0 5}$ & $\mathbf{1 0 0}$ \\
\hline
\end{tabular}

\section{-Pertenencia a Pueblos Originarios}

Dentro del grupo, el 66\% señaló pertenecer a un grupo o pueblo originario. En todos los casos sostuvieron pertenecer al pueblo mapuche, mientras que cerca de un tercio del total $(30 \%)$ indica no poseer descendencia indígena. El alto número de personas pertenecientes a grupos originarios explicaría la pertenencia cultural de la recolección de recursos en los bosques. 


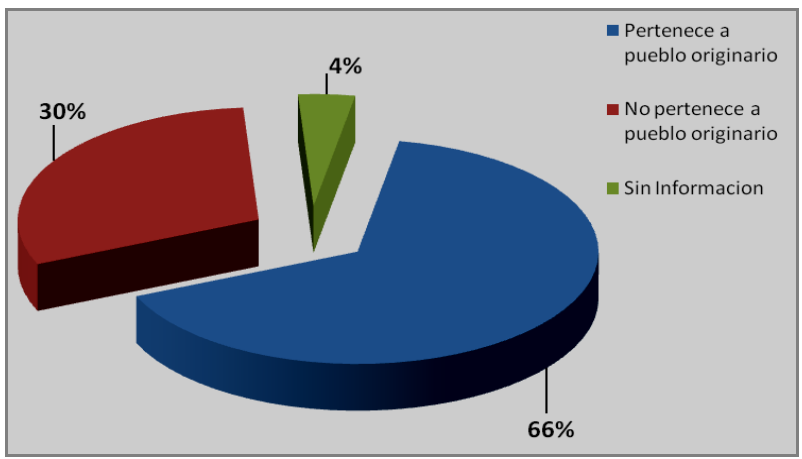

Figura 4

PERTENENCIA A PUEBLOS ORIGINARIOS DE LOS ENTREVISTADOS

\section{Antecedentes de la Actividad de Recolección}

\section{-Antigüedad en Actividades de Recolección}

Al consultar por la cantidad de tiempo (años) que tienen en actividad de recolección, se obtiene que el $67 \%$ recolecta desde hace más de cinco años. Este dato da cuenta del arraigo de esta actividad en las personas entrevistadas.

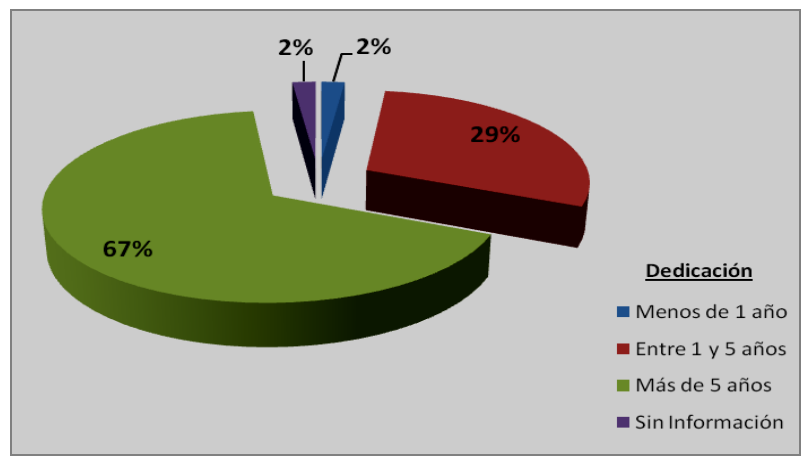

Figura $N^{\circ} 5$

\section{ANTIGÜEDAD EN ACTIVIDADES DE RECOLECCIÓN}

\section{-Aprendizaje de la Actividad}

Las personas entrevistadas señalaron que la fuente más importante de aprendizaje para esta actividad es el grupo familiar (45\%) o el ámbito de las personas cercanas. El aprendizaje resulta entonces, ser esencialmente empírico. Algunas personas mencionaron más de una forma 0 fuente de aprendizaje y, para los efectos estadísticos, se priorizó la de mayor importancia. Existe bajo grado de capacitación formal, ya sea por servicios públicos o empresas privadas (16\%), lo que radica principalmente en el desconocimiento del rubro a nivel regional y del posible potencial que se puede llegar a desarrollar. 


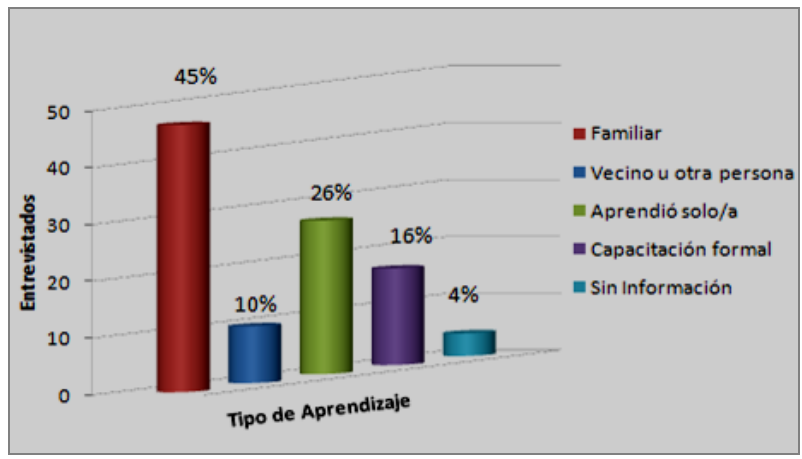

Figura $N^{\circ} 6$

APRENDIZAJE DE LA ACTIVIDAD

\section{-Capacitación}

En la Figura $N^{\circ} 6$ se aprecia que solo el 16\% de los entrevistados recibió capacitación formal para aprender esta actividad y para este caso, el origen de esta capacitación formal se puede observar en la Figura $\mathrm{N}^{\circ} 7$.

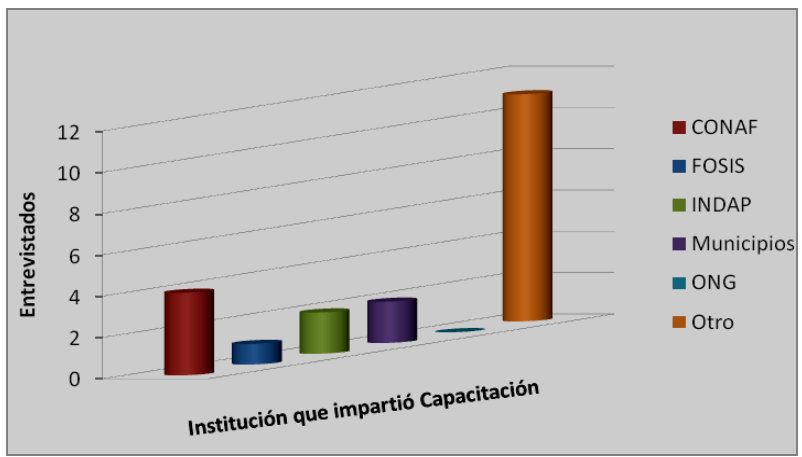

Figura $\mathrm{N}^{\circ} 7$

ORIGEN DE CAPACITACIÓN FORMAL

Un $55 \%$ del total de personas manifiesta poseer capacitación a través de la empresa regional Comercial Mañihuales Ltda. Empresa que se dedicaba principalmente a la compra de morchella y, en menor medida, rosa mosqueta y maqui. El $20 \%$ de los entrevistados manifiesta que ha participado en capacitaciones de CONAF (Corporación Nacional Forestal), institución que con la implementación de la Ley 20.283 realizó capacitaciones sobre PFNM. También se observa la participación de INDAP y Municipios (10\% cada una) a través de sus departamentos de fomento.

\section{-Participación de la Familia}

Respecto del número de personas pertenecientes al grupo familiar, que participan activamente en las actividades de recolección, el $89 \%$ sostuvo que más de un familiar se encuentra involucrado en esta actividad, que generalmente son los hijos. Esto refleja que la recolección forma parte significativa del repertorio de actividades económicas del grupo familiar y puede constituir una importante actividad conducente a la retención de la población en zonas rurales. 


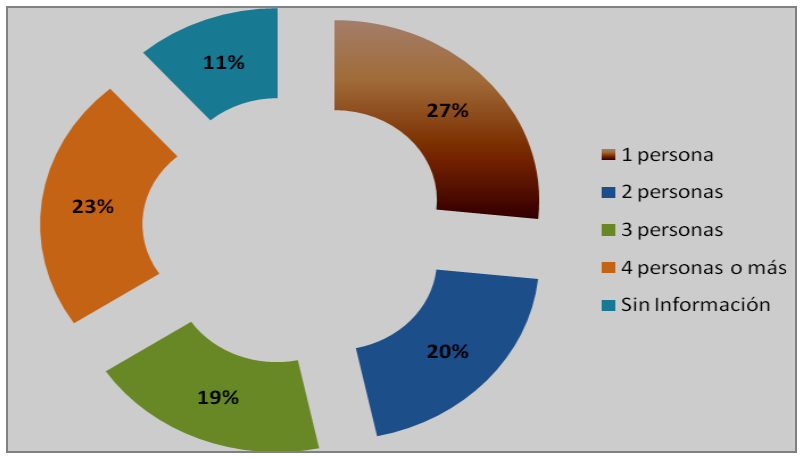

Figura $N^{\circ} 8$

NÚMERO DE PERSONAS DEL GRUPO FAMILIAR QUE PARTICIPAN EN LA RECOLECCIÓN

\section{-Gravitación en el Ingreso Familiar}

El porcentaje de los ingresos del grupo familiar derivados de la recolección resulta significativo; para un $33 \%$ de los encuestados estos ingresos corresponden a más del $21 \%$ del ingreso familiar. Sin embargo, por la naturaleza de la información que sugiere esta pregunta, hubo gran cantidad de personas (33\%) que no respondió. Cabe señalar que un $34 \%$ de los entrevistados, afirman que a través de actividades relacionadas con los PFNM generan hasta un $20 \%$ de sus ingresos mensuales, hecho que debería incrementarse en la temporada de morchella, cuando puede llegar hasta el $100 \%$.

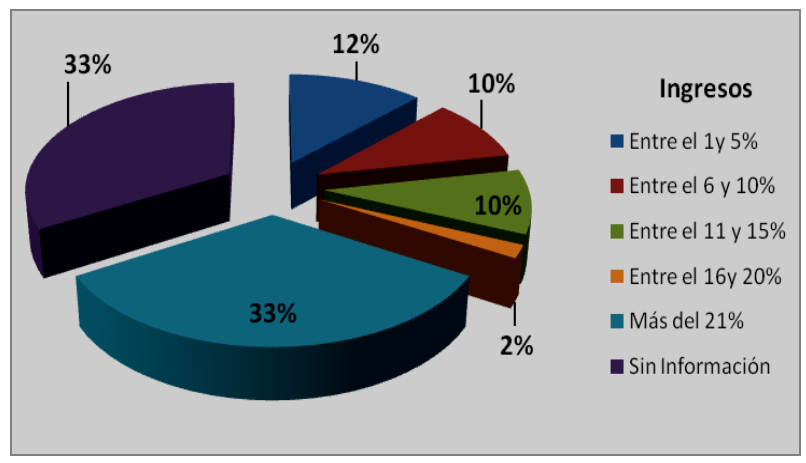

Figura $N^{\circ} 9$

PORCENTAJE DEL INGRESO FAMILIAR QUE GENERAN LAS ACTIVIDADES EN TORNO A PFNM

\section{-Productos Recolectados}

La diversidad de recursos nombrados por las personas es amplia y variada, lo que demuestra la potencialidad de esta actividad, en términos del mejoramiento de las economías familiares y principalmente de la agricultura familiar campesina.

Los recursos que cobran mayor importancia se asocian a los alimentos y a las hierbas medicinales. En las Figuras $\mathrm{N}^{\circ} 10$ a $\mathrm{N}^{\circ} 12$ se presenta algunos de los productos que en la actualidad son recolectados y aprovechados por las personas en la región. Estos productos fueron clasificados siguiendo la estructura general de los PFNM, propuesta para el país. 


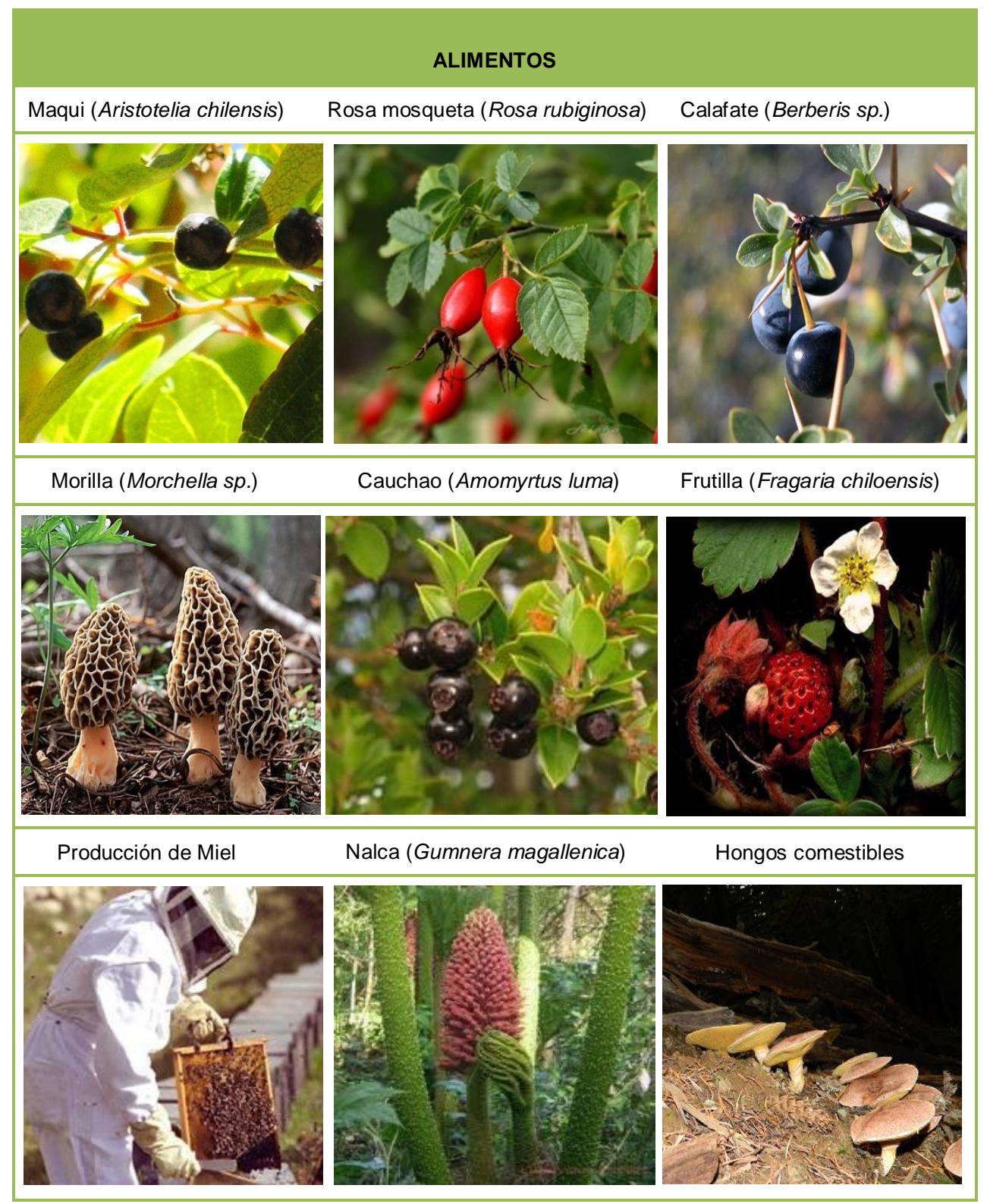

Figura $N^{\circ} 10$

PRINCIPALES PRODUCTOS ALIMENTICIOS 


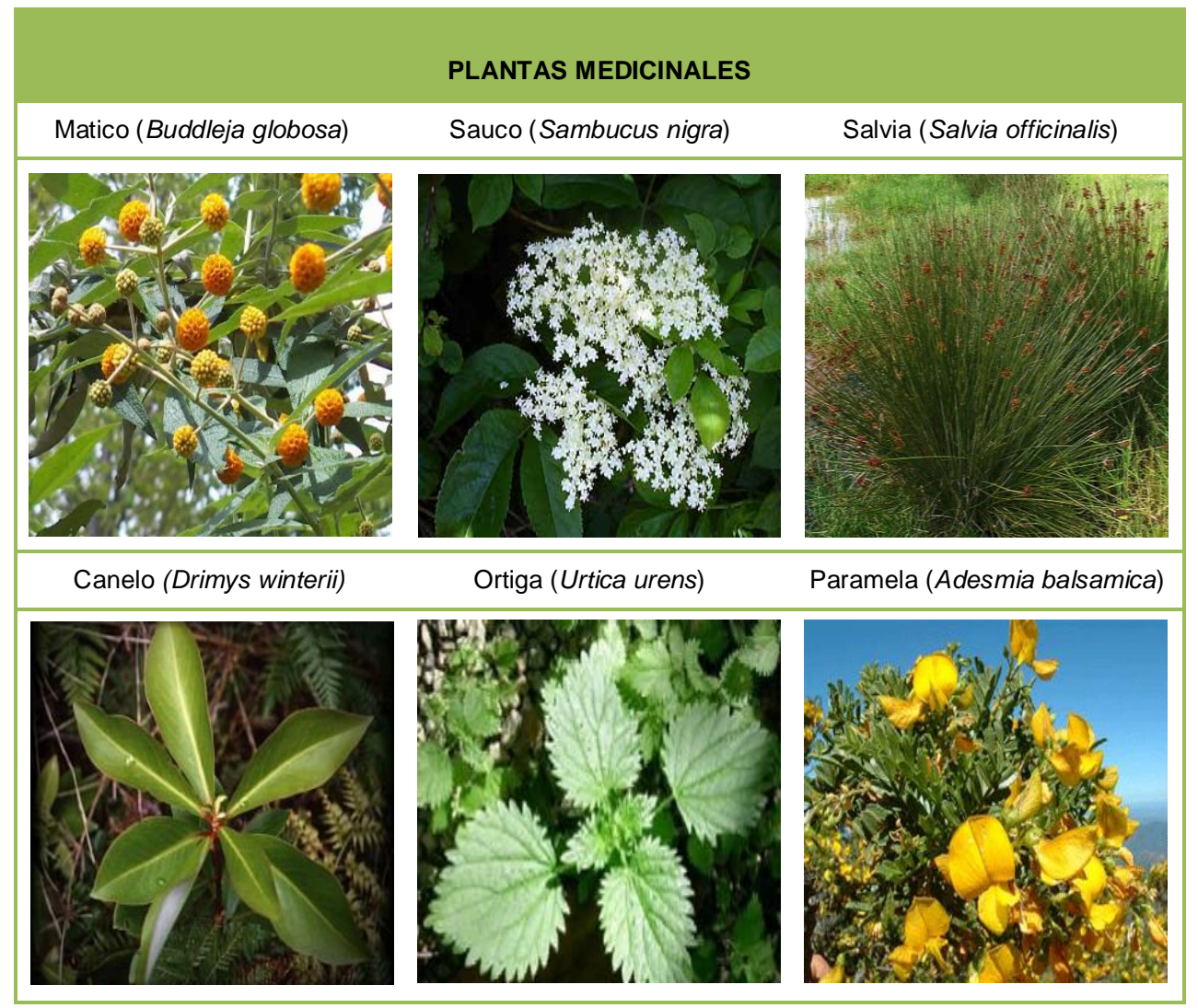

Figura $\mathrm{N}^{\circ} 11$

PRINCIPALES HIERBAS MEDICINALES 


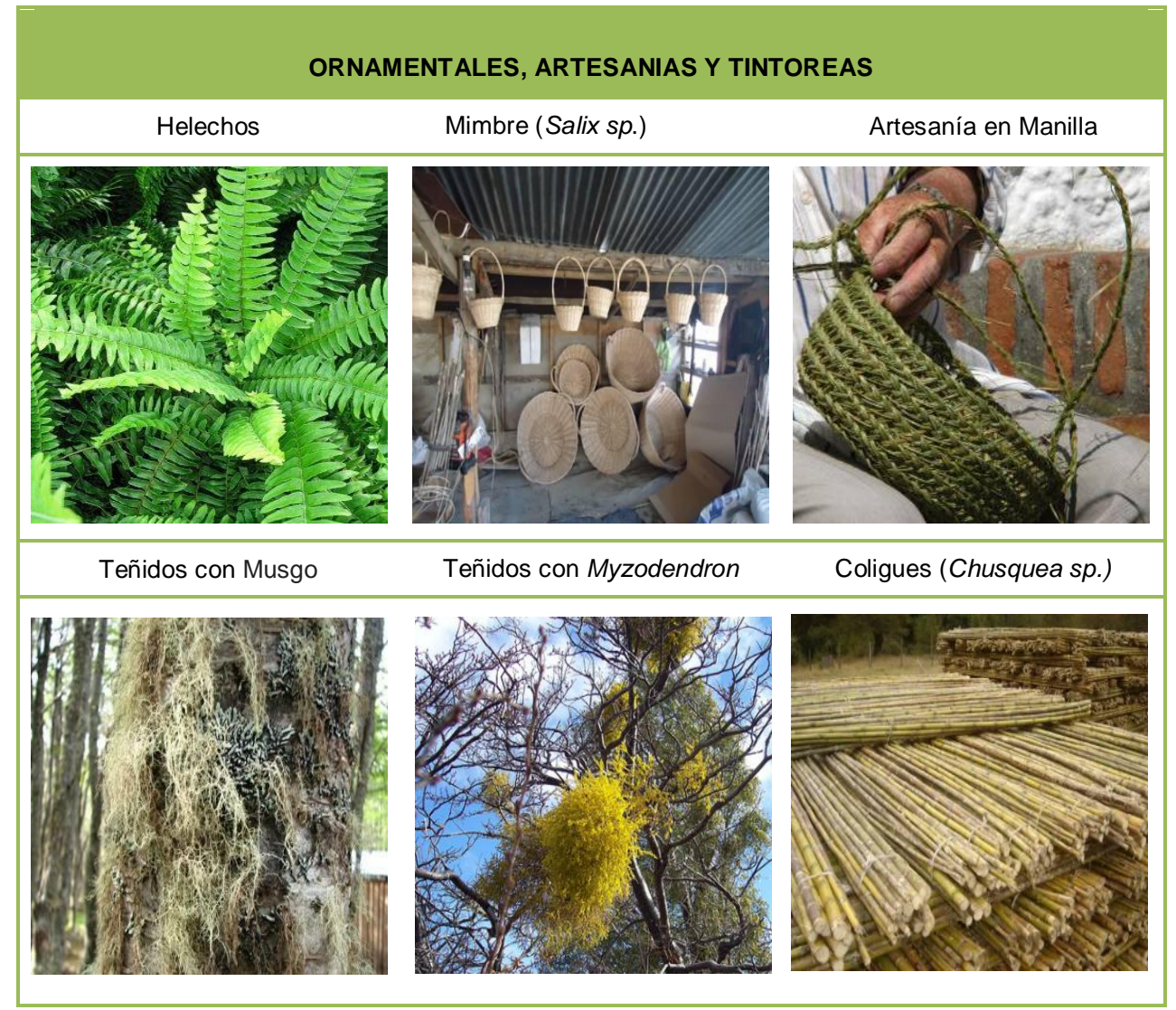

Figura $N^{\circ} 12$

ALGUNOS PRODUCTOS USADOS EN ORNAMENTACIÓN, ARTESANÍA Y TEÑIDOS.

\section{Caracterización de la Recolección}

\section{-Recolección según Producto}

Los productos que mayoritariamente se recolectan son mosqueta, calafate, morchella y maqui, con un $28,16,15$ y $11 \%$, respectivamente. La gran mayoría señaló recolectar más de un producto y fue mencionada una variedad de recursos que incluyen plantas, arbustos y hierbas medicinales típicas de la zona. No obstante, indicaron bajo el concepto de recolección algunas hierbas, frutas y hortalizas, que no corresponden a la categoría de PFNM. 


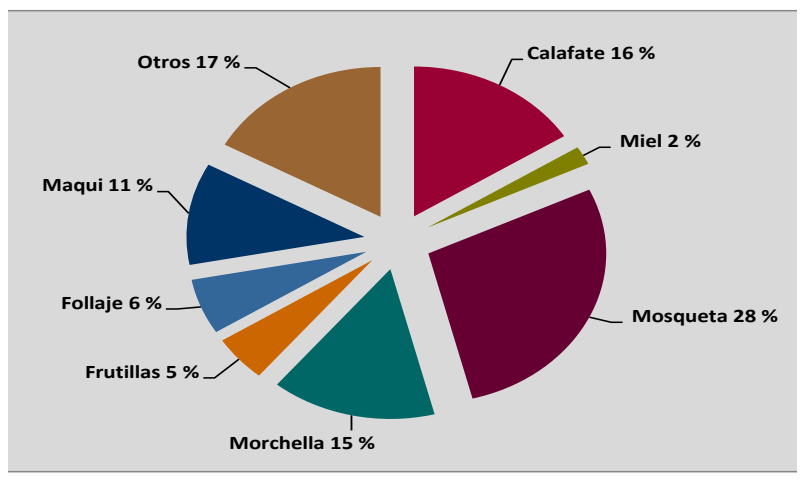

Figura $\mathrm{N}^{\circ} 13$

RECOLECCIÓN SEGÚN PRODUCTO

\section{-Productos según Localidades}

De acuerdo a las localidades donde se efectúa la recolección, existen productos principales en cada una de ellas. Destaca la presencia de maqui y follaje en la comuna de Aisén, principalmente en las localidades de Mañihuales y Valle Laguna. La rosa mosqueta se concentra en Cochrane, Río lbáñez y Cisnes, con un 30, 29 y 20\%, respectivamente.

La mayor concentración de morchella se localiza en la comuna de Cochrane (87\%), asociado a los bosques de Nothofagus spp, sin embargo, es común encontrarla en otras localidades tales como Villa Ortega, Coyhaique, Balmaceda, Guadal, Lago Verde y Tapera.

El calafate indistintamente de la especie que se trate, se distribuye en varias localidades de la región, pero es más común encontrarlo en la comuna de Río lbáñez.

Cuadro $\mathrm{N}^{\circ} 2$

RECOLECTORES SEGÚN PRODUCTO RECOLECTADO Y LOCALIDAD

\begin{tabular}{|l|c|c|c|c|c|c|c|c|}
\hline Comuna & Maqui & Calafate & Miel & Mosqueta & Morchella & Frutillas & Follaje & Otros \\
\hline Aisén & 16 & 4 & - & 4 & - & - & 11 & 13 \\
Chile Chico & 1 & 1 & - & 1 & - & 1 & - & - \\
Cisnes & 3 & 7 & - & 11 & - & 2 & - & 8 \\
Cochrane & - & 3 & - & 17 & 26 & 3 & - & 3 \\
Coyhaique & - & 1 & 1 & 5 & 3 & 4 & - & 1 \\
Río Ibáñez & - & 12 & 2 & 16 & - & - & - & 8 \\
Tortel & 1 & 1 & - & - & - & - & 1 & 2 \\
Sin Información & 2 & 3 & - & 2 & 1 & - & 1 & - \\
\hline Total & $\mathbf{2 3}$ & $\mathbf{3 2}$ & $\mathbf{3}$ & $\mathbf{5 6}$ & $\mathbf{3 0}$ & $\mathbf{1 0}$ & $\mathbf{1 3}$ & $\mathbf{3 5}$ \\
\hline
\end{tabular}

La diversidad de recursos, en relación al número de personas consultadas en cada comuna, resulta bastante similar, con la sola excepción de los recolectores de morcella que se concentran en la comuna de Cochrane.

En relación al porcentaje de participación de las localidades, destaca la comuna de Cochrane con un $26 \%$, seguida de Aisén y Rio Ibáñez con $24 \%$ y $19 \%$ respectivamente. Calafate se encontró, en mayor o menor medida, en todas las Localidades visitadas. 


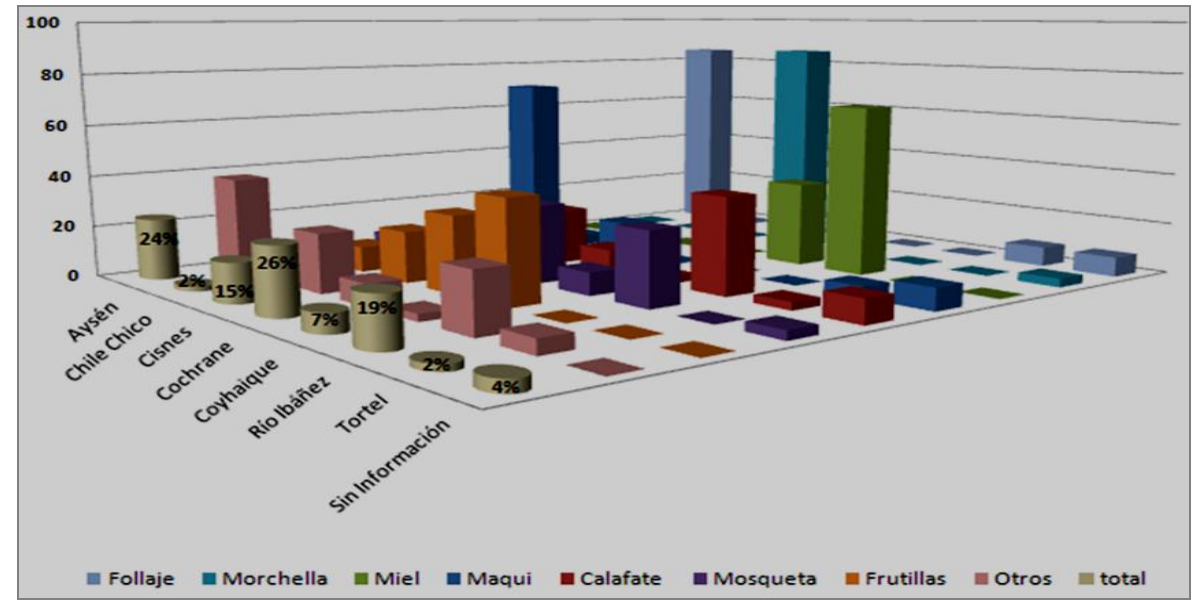

Figura $\mathrm{N}^{\circ} 14$

PRODUCTOS RECOLECTADOS SEGÚN UBICACIÓN GEOGRÁFICA (\%)

\section{-Propiedad del Lugar de Recolección según Productos}

La recolección en general se efectúa principalmente en predios pertenecientes a terceros y en predios fiscales, solo la miel se recolecta solo en terrenos propios.

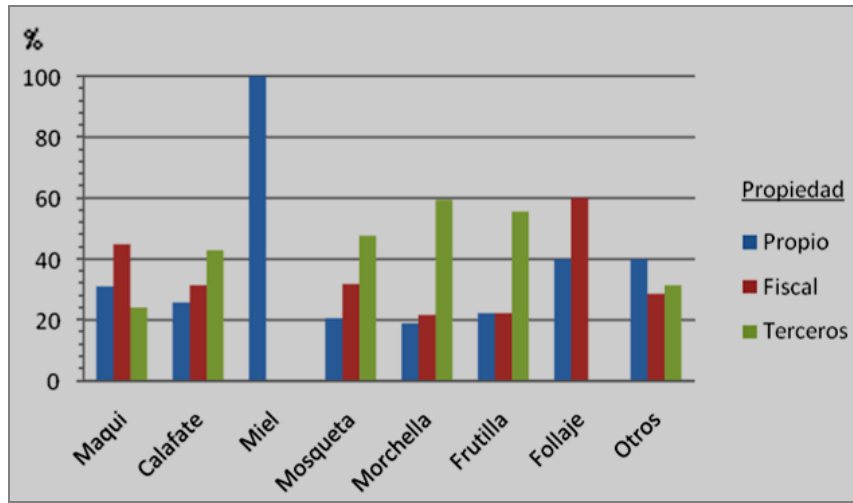

Figura $\mathrm{N}^{\circ} 15$

RECOLECCIÓN SEGÚN PROPIEDAD DE LA TIERRA (\%)

Resulta interesante señalar que del total de los entrevistados solo una persona reconoce pagar por acceder al predio en el cual recolecta, no obstante que el $72 \%$ de los predios a los que se acude para recolectar es ajeno al recolector.

\section{-Época de Colecta}

La recolección se concentra mayoritariamente entre los meses de enero y marzo. El maqui es recolectado mayoritariamente en el mes de febrero al igual que el calafate, mientras que la rosa mosqueta es recolectada en su mayoría en marzo. En el caso de morchella el mes de mayor actividad es noviembre. Ninguno de los recursos estudiados, tiene su temporada alta durante los meses de invierno. 


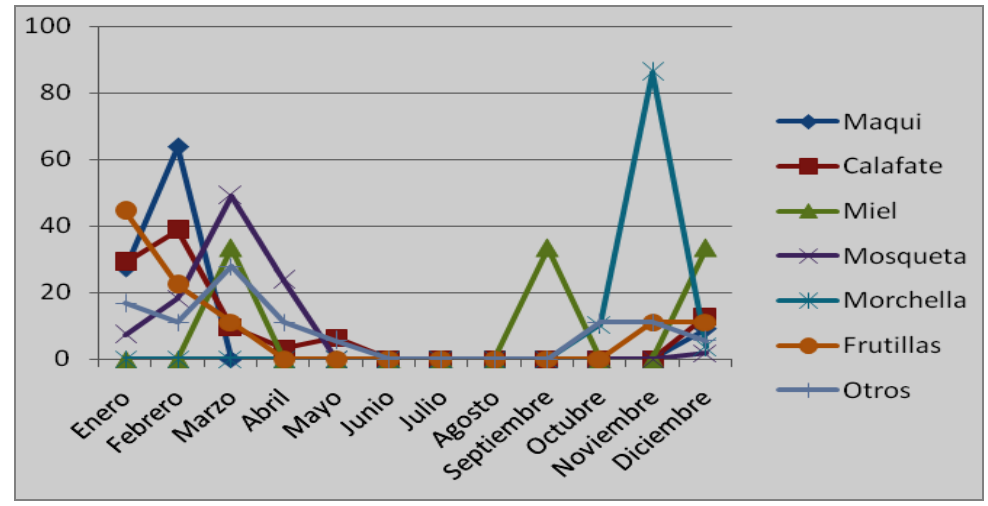

Figura $\mathbf{N}^{\circ} 16$

RECOLECCIÓN DE PRODUCTOS SEGÚN MES (\%)

\section{Antecedentes de los Productos Recolectados, Procesados y Comercializados}

\section{-Parte del Producto que se Recolecta}

En términos generales se recolecta solo una parte del producto. El $25 \%$ de los entrevistados declaran dedicarse a recolectar maqui, de ellas todos recogen sus frutos. Solo 10 recolectoras, señalan recoger además las hojas y la corteza. En la recolección de frutilla silvestre solo se aprovecha el fruto, mientras que en el calafate además del fruto se utilizan las hojas y corteza, esta última principalmente para el teñido de lanas. En la recolección de morchella las personas declaran recolectar en un $100 \%$ el cuerpo fructífero del hongo.
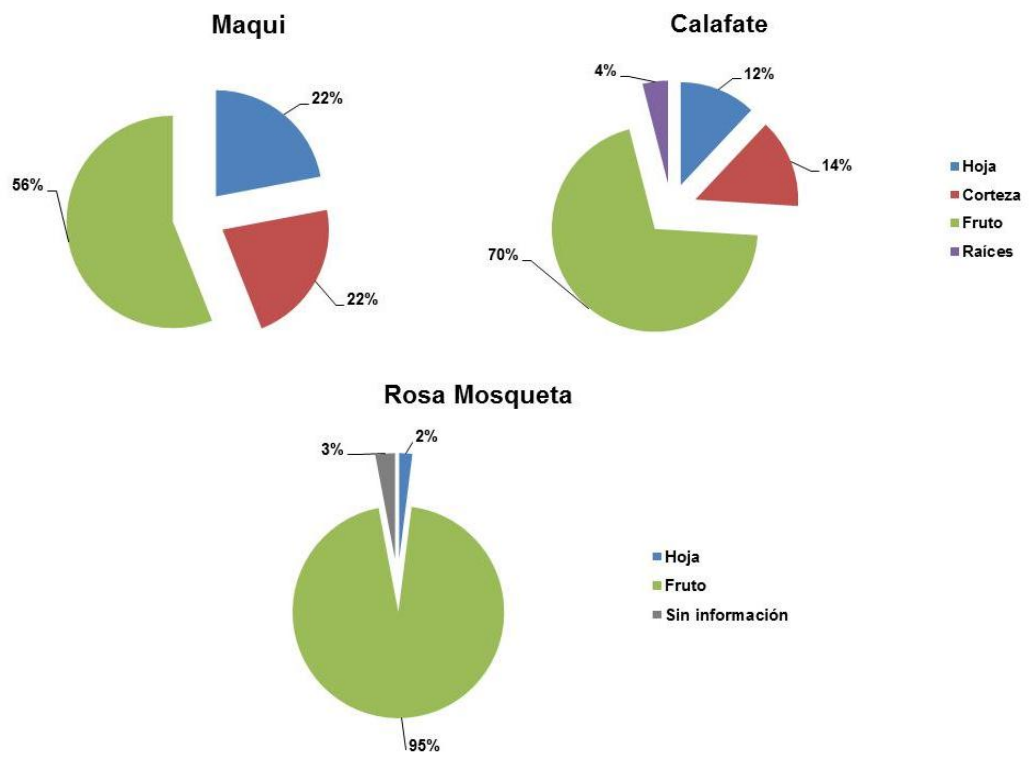

Figura $N^{\circ} 17$

PARTE DEL PRODUCTO QUE SE RECOLECTA 


\section{-Objeto de la Recolección}

En general, las personas recolectan en forma equivalente para venta y autoconsumo. Cuando venden, prefieren procesar el recurso.

Solo dos personas, ubicadas en la comuna de Cochrane, declararon ejercer como intermediarios, acopian morchella, la envían a la Región de la Araucanía y posteriormente se exporta a Europa.

Al revisar los resultados de procesamiento según recurso recolectado, se observa que un $80 \%$ del follaje está destinado al autoconsumo (arreglos florales principalmente), seguido por el maqui en donde un $54 \%$ es recolectado para el autoconsumo.

Por el contrario, en la recolección de morchella, cerca del $90 \%$ de lo recolectado se vende, es muy reducida la población que consume este hongo a nivel local.

El comercio de morchella mayoritariamente se entrega como producto fresco, sin embargo, también es entregado seco, aumentando considerablemente el precio (desde $\$ 4.000 / \mathrm{kg}$ fresco a $\$ 80.000$ - 120.000/kg deshidratado). La relación en volumen del kilogramo fresco versus deshidratado es 1:10.

El calafate y la mosqueta son productos que la gente procesa en su mayoría para elaboración de mermeladas, licor, jugos entre otros, y se emplean en forma casi equivalente para autoconsumo y para venta.

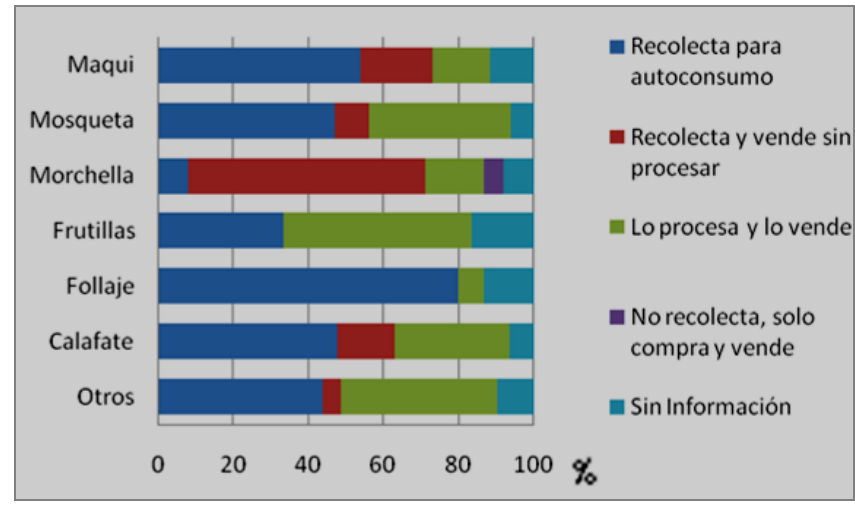

\section{Figura $\mathrm{N}^{\circ} 18$ \\ OBJETO DE LA RECOLECCIÓN SEGÚN PRODUCTO}

\section{-Tipo de Proceso según Producto}

Los datos reunidos indican que los recolectores procesan el $64 \%$ de los recursos obtenidos. El principal proceso por el cual se agrega valor es la producción de mermeladas.

Otros procedimientos corresponden a la elaboración de licores, chichas, jugos y jarabes. calafate.

El principal recurso, al que se le agrega valor, es la mosqueta. Le sigue en importancia el

El producto que posee menor procesamiento es la morchella, dado que el $62.5 \%$ de las personas que recolectan este recurso, lo comercializa sin agregar valor (fresco). 


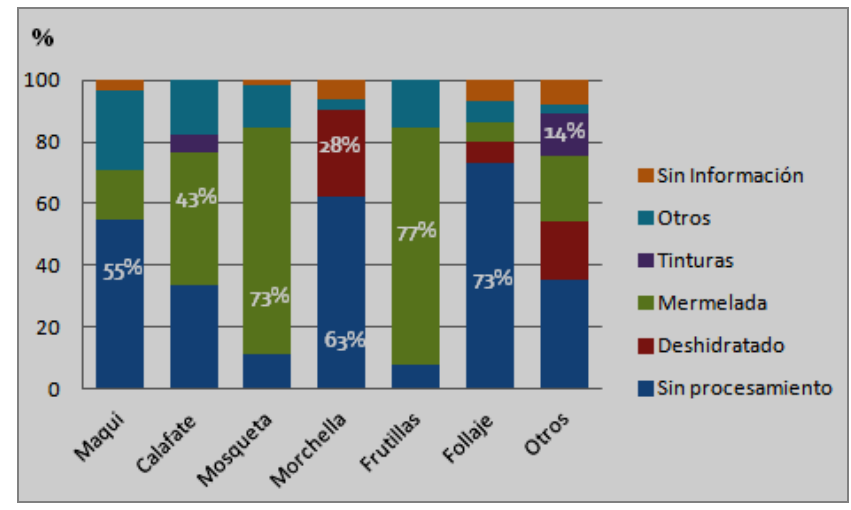

Figura $N^{\circ} 19$

TIPO Y NIVEL DE PROCESAMIENTO SEGÚN PRODUCTO

\section{-Clientes según Producto}

Los entrevistados en su mayoría indican que al momento de vender sus productos, tienen más de un comprador. Su principal opción es la venta a particulares y turistas.

Cuadro $\mathrm{N}^{\circ} 3$

TIPO DE COMPRADOR

\begin{tabular}{|l|c|c|c|c|c|c|c|c|}
\hline Cliente & Maqui & Calafate & Mosqueta & Morchella & Frutillas & Follaje & Otros & Total \\
\hline Sin comprador & 13 & 15 & 13 & 3 & 3 & 11 & 14 & 72 \\
Planta de Proceso & 2 & & & 2 & & & & 4 \\
Ferias & 1 & 3 & 4 & & & & 2 & 10 \\
Negocio & 1 & & & 1 & & & & 2 \\
Particular & 7 & 17 & 24 & 10 & 4 & 1 & 12 & 75 \\
Intermediario & & & & 17 & & & 1 & 18 \\
Turistas & 3 & 9 & 10 & & 3 & 1 & 11 & 37 \\
Otro & & 2 & & 1 & & 1 & & 4 \\
Sin Información & 1 & & 9 & 1 & 2 & & 2 & 15 \\
\hline
\end{tabular}

Se puede apreciar en el Cuadro $\mathrm{N}^{\circ} 3$ que un $32,6 \%$ de los recolectores para distintos productos indica no tener comprador, situación especialmente notoria en el caso del follaje, producto para el cual el $79 \%$ de los recolectores declara que no existe poder de compra para este recurso.

Cuadro $\mathrm{N}^{\circ} 4$

SITUACIÓN DE CLIENTES DE LOS RECOLECTORES ENTREVISTADOS.

\begin{tabular}{|l|c|c|c|}
\hline Producto & Sin comprador & Con comprador & Sin información \\
\hline Maqui & 13 & 14 & 1 \\
Calafate & 15 & 31 & 9 \\
Mosqueta & 13 & 38 & 1 \\
Morchella & 3 & 31 & 2 \\
Frutilla & 3 & 7 & \\
Follaje & 11 & 3 & $\mathbf{1 3}$ \\
Otros & 14 & 26 & \\
\hline Total & $\mathbf{7 2}$ & $\mathbf{1 5 0}$ & \\
\hline
\end{tabular}




\section{-Tipo de Venta}

Quienes señalaron comercializar maqui y sus derivados (licores, jugos, chicha, etc.), en su mayoría lo hacen al detalle. No se registró ninguna persona que indicara que su forma de venta fuera al detalle y al por mayor simultáneamente.

Morchella, es el recurso que registra más ventas al por mayor. Quienes venden de esta manera, lo hacen indistintamente a intermediarios, plantas procesadoras y a particulares. En su venta al detalle, lo cual equivale al $79 \%$ de la recolección, el comprador es en mayoritariamente un intermediario. Este recurso, reviste alto interés debido a que cuenta con un mercado que paga los mayores precios de todos los recursos estudiados y que a su vez tiene demanda a nivel de mayoristas.

Cuadro $\mathrm{N}^{\circ} 5$

TIPO DE VENTA DEL PRODUCTO

\begin{tabular}{|l|c|c|c|c|}
\hline Producto & Detalle & $\begin{array}{c}\text { Por } \\
\text { mayor }\end{array}$ & $\begin{array}{c}\text { Sin } \\
\text { venta }\end{array}$ & $\begin{array}{c}\text { Sin } \\
\text { información }\end{array}$ \\
\hline Maqui & 9 & 3 & 13 & 1 \\
Calafate & 24 & & 13 & 6 \\
Mosqueta & 34 & 1 & 15 & 7 \\
Morchella & 22 & 6 & 2 & 3 \\
Frutilla & 6 & & 3 & 3 \\
Follaje & 2 & 1 & 11 & 1 \\
Otros & 20 & 1 & 15 & 2 \\
\hline Total & $\mathbf{1 1 7}$ & $\mathbf{1 2}$ & $\mathbf{7 2}$ & $\mathbf{2 3}$ \\
\hline
\end{tabular}

\section{-Tipo de Envase}

El uso de envases para los recursos está condicionado por su procesamiento. Las cifras indican claramente que para el caso de la mosqueta, al ser transformada en mermelada, se utiliza el tradicional envase de vidrio. El calafate también se procesa preferentemente como mermelada e igualmente se la envasa en vidrio. Para la morchella, en tanto se emplea bolsas de plástico. El maqui es recolectado en tarros plásticos y envasado en sacos.

\section{Cuadro $\mathrm{N}^{\circ} 6$}

TIPO DE ENVASES UTILIZADOS PARA LA CONSERVACIÓN Y VENTA DE PRODUCTOS

\begin{tabular}{|l|c|c|c|c|c|c|c|c|c|}
\hline Producto & $\begin{array}{c}\text { Bolsa } \\
\text { plástica }\end{array}$ & $\begin{array}{c}\text { Bolsa } \\
\text { papel }\end{array}$ & $\begin{array}{c}\text { Envase } \\
\text { vidrio }\end{array}$ & $\begin{array}{c}\text { Caja } \\
\text { cartón }\end{array}$ & $\begin{array}{c}\text { Caja } \\
\text { madera }\end{array}$ & Saco & $\begin{array}{c}\text { Envase } \\
\text { plástico }\end{array}$ & Otro & $\begin{array}{c}\text { Sin } \\
\text { inf. }\end{array}$ \\
\hline Maqui & 4 & & 8 & & & 1 & 7 & 2 & 4 \\
Calafate & 6 & & 23 & & & 2 & 6 & 3 & 1 \\
Mosqueta & 6 & & 44 & 1 & 1 & 2 & 2 & 1 & 4 \\
Morchella & 16 & 2 & & 1 & 1 & 4 & & 8 & 3 \\
Frutillas & 1 & & 7 & & & & 1 & & 3 \\
Follaje & 2 & 1 & 1 & & & & 9 & & 1 \\
Otros & 5 & 3 & 13 & & & 1 & 9 & 2 & 2 \\
\hline Total & 40 & $\mathbf{6}$ & $\mathbf{9 6}$ & $\mathbf{2}$ & $\mathbf{2}$ & $\mathbf{1 0}$ & $\mathbf{3 4}$ & $\mathbf{1 6}$ & $\mathbf{1 8}$ \\
\hline
\end{tabular}

\section{-Precios}

El maqui y el calafate son comercializados preferentemente como fruto a granel, como mermelada y como licor.

La miel es procesada y vendida a compradores particulares, al detalle, en envases de plástico y vidrio.

La mosqueta se vende generalmente por kilogramo de fruto a granel, también procesado 
como mermelada o bien en pulpa. Las frutillas son procesadas en su mayoría como mermeladas.

Respecto del follaje no se dispone de información suficiente en este aspecto, lo cual resulta coherente con el hecho que este recurso prácticamente no se comercializa.

Cuadro $\mathrm{N}^{\circ} 7$

PRECIO DE PRODUCTOS SEGÚN FORMATO DE VENTA

\begin{tabular}{|c|c|c|c|c|c|c|}
\hline Producto & $\begin{array}{c}\text { Fruto granel } \\
\qquad(\$ / k g)\end{array}$ & $\begin{array}{c}\text { Mermelada } \\
(\$ / \mathbf{k g})\end{array}$ & $\begin{array}{c}\text { Licor } \\
(\$ 700 / c c) \\
\end{array}$ & $\begin{array}{c}\text { Miel } \\
(\$ / \mathbf{k g})\end{array}$ & $\begin{array}{c}\text { Hongo fresco } \\
(\$ / \mathbf{k g})\end{array}$ & $\begin{array}{c}\text { Hongo } \\
\text { deshidratado } \\
(\$ / \mathbf{k g})\end{array}$ \\
\hline $\begin{array}{l}\text { Maqui } \\
\text { Calafate } \\
\text { Miel } \\
\text { Mosqueta } \\
\text { Morchella } \\
\text { Frutillas }\end{array}$ & $\begin{array}{l}500-3.500 \\
500-1.500 \\
500-1.000 \\
2.000-3.000\end{array}$ & $\begin{array}{l}5.000-6.000 \\
6.000 \\
2.000-5.500 \\
5.000-6.000\end{array}$ & $\begin{array}{l}2.000-3.500 \\
4.000 \\
2.000-3.500\end{array}$ & $2.000-3.000$ & $2.000-10.000$ & $80.000-120.000$ \\
\hline
\end{tabular}

\section{-Disponibilidad del Recurso}

Respecto de la disponibilidad de los recursos para recolección, los entrevistados perciben mayoritariamente que estos se han mantenido en el tiempo, su opinión deriva de su contacto directo con los recursos y corresponde a una apreciación global de todos los recursos simultáneamente.

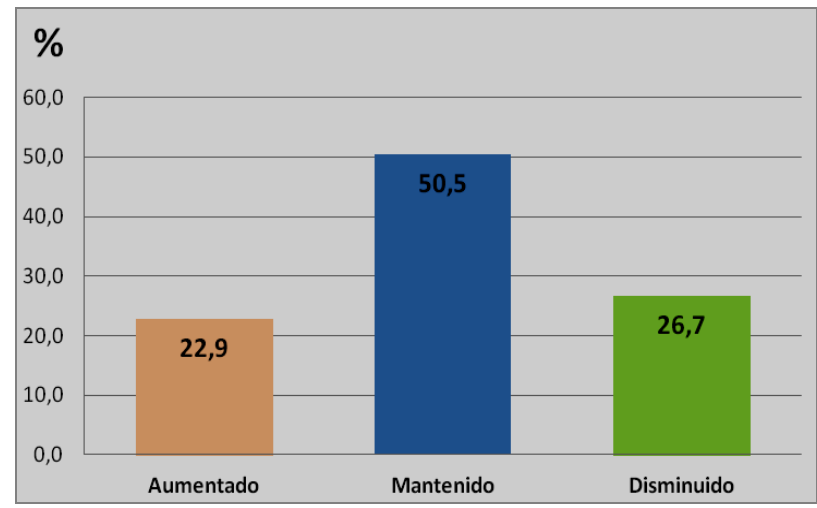

Figura $\mathrm{N}^{\circ} 20$

PERCEPCIÓN SOBRE LA DISPONIBILIDAD DE LOS RECURSOS

Cuando se consultó a los entrevistados por las razones que explican la disponibilidad de los recursos en el tiempo, responden señalando más de una opción para explicar estos cambios en los casos que consideran que ha variado.

La mayoría de personas (48\%) no mantiene información del estado actual de los recursos o no sabe. Este hecho, responde a una asimetría de información en el rubro de los PFNM. Solo el $13 \%$ atribuye disminuciones a mayor presión sobre el recurso. 


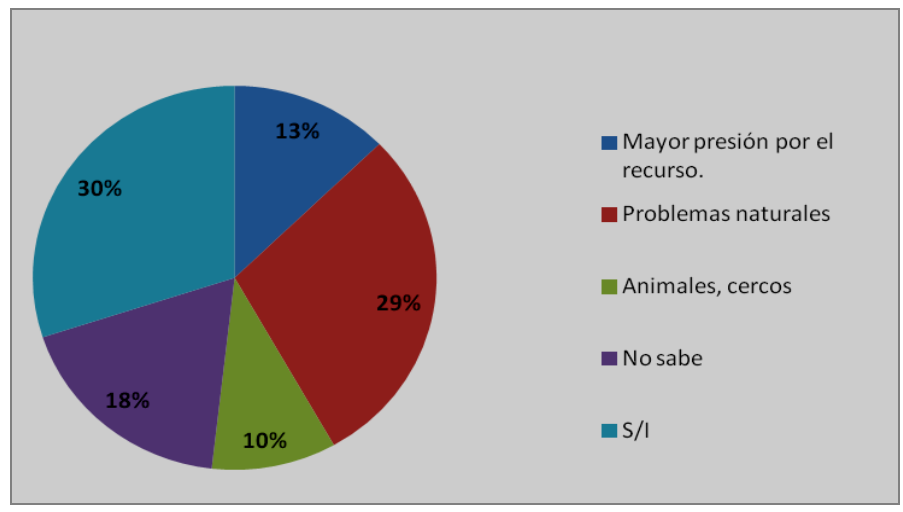

RAZONES QUE EXPLICAN LA DISPONIBILIDAD DE LOS RECURSOS

\section{-Necesidades de los Recolectores para Comercializar sus Productos}

Ante la consulta sobre los aspectos de su actividad que debieran potenciarse para impulsar sus ventas, señalaron en primer término el acceso a los mercados, seguido de la necesidad de contar con capacitación específica para sus requerimientos y finalmente aspectos de infraestructura y asociatividad.

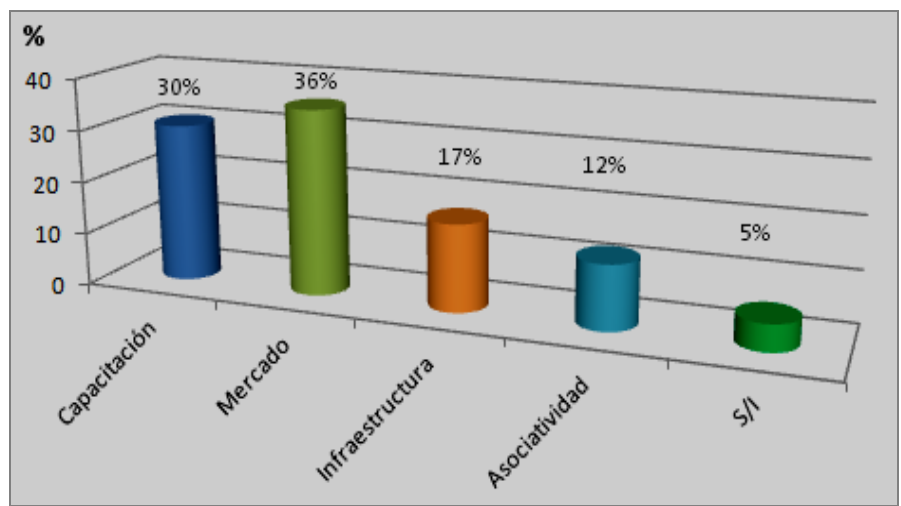

Figura $N^{\circ} 22$

NECESIDADES PARA AUMENTAR CAPACIDAD DE VENTA

\section{CONCLUSIONES}

En la Patagonia chilena la recolección de PFNM constituye una actividad de gran tradición y arraigo entre las familias rurales. Si bien es de pequeña escala y desarrollada con métodos artesanales, constituye una práctica relevante en el contexto de la economía de las personas entrevistadas.

La actividad de recolección, dentro de la diversidad de actividades que se efectúa en el espacio rural, es esencialmente femenina. El estudio indicó que el $87 \%$ de la participación en la recolección está representada por mujeres. 
Los jóvenes menores de 30 años en su mayoría no se dedican a la actividad de recolección, el $80 \%$ de los recolectores se encuentra entre los 30 y 60 años.

Existe una percepción de proyección de la actividad de recolección en la región.

El grado de escolaridad de los recolectores es bajo, cerca del $60 \%$ de los entrevistados ha cursado solo educación básica. Esto indica la necesidad de generar programas de capacitación específica en el tema.

Más del $60 \%$ de los entrevistados dice pertenecer a una etnia originaria y cerca del $50 \%$ menciona que el aprendizaje de la actividad lo adquirió en el ámbito familiar. La importancia de esta actividad en el ingreso familiar, se refleja en que un 34\% de los entrevistados menciona que más del $20 \%$ de sus ingresos proviene de actividades ligadas a los PFNM, y puede llegar en épocas del año al $100 \%$.

|El recurso que reviste mayor interés en términos económicos es la morchella, aunque su recolección se encuentra acotada a sectores geográficos limitados. La mosqueta, el maqui y calafate resultan interesantes dada su amplia cobertura geográfica, arraigo de la práctica de su recolección y posibilidades de venta y aumento de demanda externa.

Es necesario tener presente que los predios de terceros y los predios fiscales constituyen los principales sectores en donde se desarrolla la recolección, lo cual podría tornarse en un factor de fragilidad ante una propuesta de crecimiento de la actividad.

Debido al carácter tradicional de la actividad y la forma rústica de tratar los recursos, la recolección de los productos estudiados presenta múltiples deficiencias. Los procesos de agregación de valor son simples, en tanto que la comercialización se efectúa dentro del marco de la economía informal, con acceso restringido a los mercados.

\section{REFERENCIAS}

FAO, 1999. Hacia una definición uniforme de los Productos forestales no madereros. UNASYLVA 50(198): 63-64.

INFOR, 2014. Productos Forestales no Madereros (PFNM). Boletín № 19. Santiago. Chile. 4pp. (En edición) 
